\title{
Dynamically screened Coulomb interaction in the parent compounds of hole-doped cuprates: Trends and exceptions
}

\author{
F. Nilsson, ${ }^{1, *}$ K. Karlsson, ${ }^{2}$ and F. Aryasetiawan ${ }^{1}$ \\ ${ }^{1}$ Department of Physics, Division of Mathematical Physics, Lund University, Professorsgatan 1, SE-223 63 Lund, Sweden \\ ${ }^{2}$ Department of Engineering Sciences, University of Skövde, SE-541 28 Skövde, Sweden
}

(Received 20 December 2018; published 19 February 2019)

\begin{abstract}
Although cuprate high-temperature superconductors were discovered already in 1986 the origin of the pairing mechanism remains elusive. While the doped compounds are superconducting with high transition temperatures $T_{c}$, the undoped compounds are insulating due to the strong effective Coulomb interaction between the $\mathrm{Cu} 3 d$ holes. We investigate the dependence of the maximum superconducting transition temperature $T_{c} \max$ on the on-site effective Coulomb interaction $U$ using the constrained random-phase approximation. We focus on the commonly used one-band model of the cuprates, including only the antibonding combination of the $\mathrm{Cu} d_{x^{2}-y^{2}}$ and $\mathrm{O} p_{x}$ and $p_{y}$ orbitals and find a screening-dependent trend between the static value of $U$ and $T_{c \max }$ for the parent compounds of a large number of hole-doped cuprates. Our results suggest that superconductivity may be favored by a large on-site Coulomb repulsion. We analyze both the trend in the static value of $U$ and its frequency dependence in detail and, by comparing our results to other works, speculate on the mechanisms behind the trend.
\end{abstract}

DOI: 10.1103/PhysRevB.99.075135

\section{INTRODUCTION}

One of the most important experimental insights about the high-temperature copper oxide superconductors is how $T_{c}$ is correlated with the materials structure expressed as a function of doping, pressure, and compositions. Phase diagrams of the high- $T_{c}$ cuprates as a function of doping concentration reveal a generic feature common to all compounds, showing the characteristic parabolic curve separating the superconducting and normal phases. The crystal structure of the cuprates exhibits the generic copper oxide planes where the dominant low-energy physics is believed to be constrained. It has been known for a long time that $T_{c \text { max }}$ increases with the number of $\mathrm{CuO}_{2}$ layers and for a given number of layers there is a strong dependence of $T_{c \text { max }}$ on the cuprate family. It was, however, not known at the microscopic level on which quantum mechanical parameters $T_{c \text { max }}$ depended. This puzzle was investigated and analyzed in detail by Pavarini et al. more than a decade ago, and they found an interesting and important trend showing a correlation between $T_{c \max }$ and the hopping parameters [1]. Thorough investigation of the phase diagrams of the high- $T_{c}$ cuprates, on the other hand, has revealed that the macroscopic properties of the copper oxides are decisively influenced by strong electron-electron interaction (large Hubbard $U$ ) between the copper $3 d$ holes (see, e.g., Ref. [2]). The large Coulomb repulsion also profoundly influences other fundamental properties which do not follow the standard Fermiliquid theory, which is exhibited already in the case of zero doping in which the material becomes an antiferromagnetic Mott insulator. While a large Coulomb repulsion was at first thought not to be conducive for the formation of Cooper pairs leading to superconductivity, theoretical studies of the

\footnotetext{
*fredrik.nilsson@teorfys.lu.se
}

two-dimensional single-band Hubbard model have indicated that superconductivity can be favored by a large $U$ [3-7].

The Heisenberg nearest-neighbor exchange parameter $J$ is a quantity that is intimately related to the Hubbard $U$. In the large- $U$ limit the quantities are directly related as $J=-4 t^{2} / U$, where $t$ is the nearest-neighbor hopping. In a recent experimental study [8] Mallet et al. investigated the dependence of $J$ on $T_{c}$ max for the systems $R(\mathrm{Ba}, \mathrm{Sr})_{2} \mathrm{Cu}_{3} \mathrm{O}_{y}$. It was shown that $J$ had a strong correlation with $T_{c \max }$ for the considered compounds. However, it was also shown that changing internal pressure by ion substitution and varying the external pressure have identical effects on $J$ but opposite effects on $T_{c \text { max }}$. On the other hand, the refractivity sum was shown to have a strong correlation with $T_{c \max }$, which led the authors to suggest a dielectric rather than a magnetic pairing mechanism.

The purpose of the present work is to delve deeper into the microscopic origin of the trend in $T_{c \max }$ by studying its dependence on the strength of the Coulomb repulsion, or Hubbard $U$. Although $U$ and $J$ are directly related in the limit where $U$ is much larger than the bandwidth, for the cuprate compounds $U$ is of the same order as the bandwidth $[9,10]$, and hence, this relation is not guaranteed to hold. Further on, the value of $U$ is directly influenced by the dielectric screening, and an investigation of the material dependence of $U$ could therefore be a route to understanding the correlation between $T_{c \max }$ and the refractivity sum reported in Ref. [8].

We compute the Hubbard $U$ using the constrained randomphase approximation (cRPA) [11,12] as implemented in the full-potential linearized augmented plane-wave (FLAPW) codes FLEUR and SPEX $[13,14]$. The cRPA yields both the static (time-averaged) value and the full frequency dependence of $U$ and allows for a detailed analysis of the screening channels responsible for renormalizing the bare Coulomb interaction $v$. 
We consider a wide range of hole-doped cuprate compounds starting with the well-studied $\mathrm{La}_{2} \mathrm{CuO}_{4}$ as well as $\mathrm{TlBa}_{2} \mathrm{CuO}_{6}$ and $\mathrm{HgBa}_{2} \mathrm{CuO}_{4}$ and continuing with the compounds $R(\mathrm{Ba}, \mathrm{Sr})_{2} \mathrm{Cu}_{3} \mathrm{O}_{6}(R=\mathrm{Y}, \mathrm{Yb}, \mathrm{Nd}, \mathrm{La})$ that were also studied experimentally in Ref. [8]. In the latter compounds, changing the ion size yields a change in size of the unit cell and can therefore be considered a change in the "internal pressure" of the compound [8]. We also explore the effects of external pressure on $U$ by systematically changing the lattice parameters.

With the exception of $\mathrm{La}_{2} \mathrm{CuO}_{4}$, we find a screeningdependent correlation between $T_{c \text { max }}$ and both the static value of $U$ and the ratio $U / t$, suggesting that superconductivity in the cuprates may be favored by a large on-site Coulomb repulsion. In contrast to $J$ we also find that external and internal pressures have the same effect on $U$; that is, $U$ increases with both internal and external pressure. However, the increase is not sufficiently large to account for the observed increase in $T_{c \text { max }}$. In the numerical studies in Refs. $[15,16]$ it was found that superconductivity was favored by a small charge-transfer energy $\left(\epsilon_{d}-\epsilon_{p}\right)$. Together with the trend in $U$ in the present paper this suggests that superconductivity may be favored by having a large $U$ and small charge-transfer energy, which would lead to a charge-transfer insulating parent compound with the lower Hubbard band below the $O p$ states. Since $\mathrm{La}_{2} \mathrm{CuO}_{4}$ has both a large $U$ and a large charge-transfer energy, this could offer an explanation of why $\mathrm{La}_{2} \mathrm{CuO}_{4}$ does not follow the trend in $U$.

We also consider the full frequency-dependent $U(\omega)$ for these compounds. We analyze the different screening channels and show that the $p-d$ screening channel, which gives rise to peaks around $8-9 \mathrm{eV}$ in all cuprate compounds, is much stronger in $\mathrm{La}_{2} \mathrm{CuO}_{4}$ than in the other compounds. In that sense $\mathrm{La}_{2} \mathrm{CuO}_{4}$ is an unusual case and may not be a good representative prototype for a general cuprate compound. Furthermore, we show that $U$ is highly material dependent, suggesting that the common assumption of using the same value of $U$ for all cuprate compounds can yield misleading conclusions.

\section{METHOD}

\section{A. cRPA}

To study the materials dependence of the Hubbard $U$, we use the cRPA method [11,12]. In the cRPA method, the screening channels expressed in terms of the polarizations are decomposed into those within the model $\left(P_{d}\right)$ and the rest $\left(P_{r}\right)$ :

$$
P=P_{d}+P_{r} .
$$

It can then be shown that the effective Coulomb interaction among the electrons residing in the model subspace (the $d$ subspace) is given by

$$
U(\omega)=\left[1-v P_{r}(\omega)\right]^{-1} v
$$

This effective interaction is physically interpreted as the Hubbard $U$, which is now a function of frequency. This interpretation is based on the fact that when $U$ is screened by the polarization $P_{d}$ of the model, one obtains the fully screened interaction:

$$
W(\omega)=[1-v P(\omega)]^{-1} v=\left[1-U(\omega) P_{d}(\omega)\right]^{-1} U(\omega) .
$$

In practice the polarization is computed from the localdensity approximation (LDA) band structure [17] within the random-phase approximation (RPA), which for a given spin is given by

$$
\begin{aligned}
P\left(\mathbf{r}, \mathbf{r}^{\prime} ; \omega\right)= & \sum_{\mathbf{k} n}^{\text {occ }} \sum_{\mathbf{k}^{\prime} n^{\prime}}^{\text {unocc }} \frac{\psi_{\mathbf{k} n}^{*}(\mathbf{r}) \psi_{\mathbf{k}^{\prime} n^{\prime}}(\mathbf{r}) \psi_{\mathbf{k}^{\prime} n^{\prime}}^{*}\left(\mathbf{r}^{\prime}\right) \psi_{\mathbf{k} n}\left(\mathbf{r}^{\prime}\right)}{\omega-\epsilon_{\mathbf{k}^{\prime} n^{\prime}}+\epsilon_{\mathbf{k} n}+i \delta} \\
& -\frac{\psi_{\mathbf{k} n}(\mathbf{r}) \psi_{\mathbf{k}^{\prime} n^{\prime}}^{*}(\mathbf{r}) \psi_{\mathbf{k}^{\prime} n^{\prime}}\left(\mathbf{r}^{\prime}\right) \psi_{\mathbf{k} n}^{*}\left(\mathbf{r}^{\prime}\right)}{\omega+\epsilon_{\mathbf{k}^{\prime} n^{\prime}}-\epsilon_{\mathbf{k} n}-i \delta}
\end{aligned}
$$

In the LDA the conduction band in the cuprates originates from the antibonding combination of $\mathrm{Cu} d_{x^{2}-y^{2}}$ with the oxygen $p_{x} / p_{y}$ orbitals and has $d_{x^{2}-y^{2}}$ symmetry. The bonding and nonbonding bands, commonly referred to as the $\mathrm{O} p_{x / y}$ bands, are located around $6 \mathrm{eV}$ below the Fermi energy. Commonly used models for the cuprates include (i) the one-band model derived from the antibonding conduction band, (ii) the twoband model that apart from the conduction band includes the narrow band just below the Fermi energy originating from the hybridization between the $\mathrm{Cu} 3 d_{z^{2}}$ and apex oxygen $p_{z}$ orbitals, (iii) the three-band model that includes the antibonding conduction band as well as the bonding and nonbonding combinations, and (iv) the four-band model that includes all the above-mentioned bands. In this work we focus on the one-band model since it provides the minimal low-energy model of the cuprates.

To define the $d_{x^{2}-y^{2}}$ model subspace we use maximally localized Wannier functions (MLWFs) [18-20] that are derived from the LDA band structure. Hence, $P_{d}$ in Eq. (1) is the polarization within the $d_{x^{2}-y^{2}}$ conduction band. Since the $d_{x^{2}-y^{2}}$ band is not isolated, we use the disentanglement approach [21] to get a well-defined one-particle band structure and model polarization. In this method the hybridization between the model and the rest is cut in the Hamiltonian

$$
\tilde{H}=\left(\begin{array}{cc}
H_{d d} & 0 \\
0 & H_{r r}
\end{array}\right) .
$$

The $r$ subspace polarization is then calculated as $P_{r}=P-P_{d}$, where the full polarization $P$ and the $d$ subspace polarization $P_{d}$ are calculated for the disentangled band structure according to Eq. (4). In Fig. 1 we show the Wannier
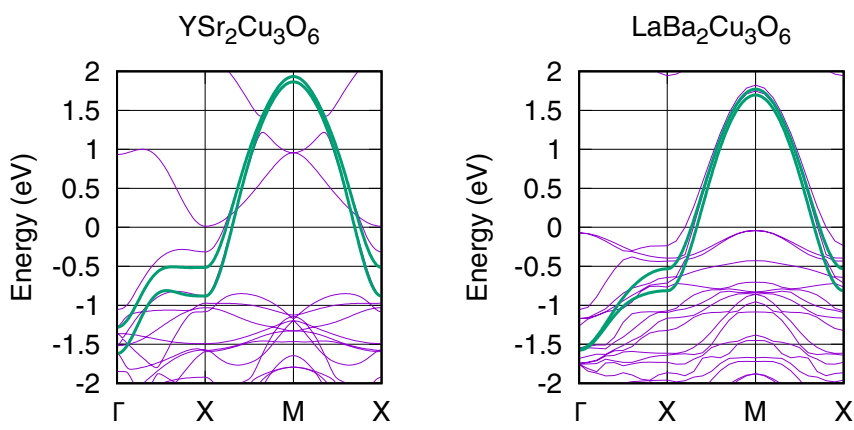

FIG. 1. LDA and Wannier interpolated band structures of $\mathrm{YSr}_{2} \mathrm{Cu}_{3} \mathrm{O}_{6}$ and $\mathrm{LaBa}_{2} \mathrm{Cu}_{3} \mathrm{O}_{6}$. The Fermi energy was set to zero. 
interpolated band structures for the two compounds $\mathrm{YSr}_{2} \mathrm{Cu}_{3} \mathrm{O}_{6}$ and $\mathrm{LaBa}_{2} \mathrm{Cu}_{3} \mathrm{O}_{6}$.

We compute $U$ only for the undoped parent compounds. However, since the metallic screening from within the $d_{x^{2}-y^{2}}$ conduction band is removed within the cRPA, $U$ is expected to be very weakly dependent on the doping. Hence, the values of $U$ for the parent compounds can also be used in the doped cases.

\section{B. Simulation of external pressure}

We consider the effect of external pressure for the compound $\mathrm{YbBa}_{2} \mathrm{Cu}_{3} \mathrm{O}_{6}$ by scaling the lattice parameters. With the exception of $\mathrm{La}_{2} \mathrm{CuO}_{4}$, where the effect of pressure is approximately isotropic [22,23] (i.e., the $a$ and $c$ lattice parameters are scaled by the same factor), a generic feature of the cuprates seems to be that the effect of hydrostatic pressure on the $c$ lattice parameter is around twice as big as that of the in-plane lattice parameter $a$ [23]. Therefore, we approximate hydrostatic pressure by scaling $a$ with $x \%$ and $c$ with $2 x \%$. Experimentally, $T_{c}$ max increases with moderately applied pressure and decreases again at high pressure (larger than 5-7 GPa) [23,24]. In Ref. [25] $c$ was shown to decrease about $4 \%$, and $a$ was shown to decrease about $2 \%$ with a pressure of $6 \mathrm{GPa}$ for $\mathrm{HgBa}_{2} \mathrm{Ca}_{2} \mathrm{Cu}_{3} \mathrm{O}_{8+\delta}$. Therefore, we consider scalings of $a$ and $c$ below these numbers in this work.

In Ref. [26] both the uniaxial and hydrostatic pressure derivatives of $T_{c}$ were determined for $\mathrm{HgBa}_{2} \mathrm{CuO}_{4+\delta}$ close to the optimal doping level. It was found that $T_{c}$ increases with a decreasing unit cell area of the $\mathrm{Cu}-\mathrm{O}$ planes as well as with an increasing separation of the planes. To investigate the effect of uniaxial pressure on $U$ we also consider scaling only $a$, which corresponds to applying only in-plane pressure.

\section{COMPUTATIONAL DETAILS}

We use the LDA band structure calculated with the FLAPW code FLEUR [14] as a starting point. The MLWFs were computed using the WANNIER90 library [18-20], and $U$ was computed within the cRPA as implemented in the SPEX code $[13,14]$. We only considered spin polarization for the compounds $\mathrm{YbBa}_{2} \mathrm{Cu}_{3} \mathrm{O}_{6}$ and $\mathrm{NdBa}_{2} \mathrm{Cu}_{3} \mathrm{O}_{6}$ since the other compounds are not spin polarized within the LDA. All calculations were converged with respect to the FLAPW basis set, the number of bands used to compute the polarization function, the number of $\mathbf{k}$ points used in the LDA, and the cRPA calculation. For example, this required the use of between 300 and 400 bands in the computation of the polarization function for the different compounds.

The bands used to construct the Wannier functions were defined using an energy window where for each $\mathbf{k}$ point all states with an energy inside the energy window were used in the Wannier function construction. In Table I we present the energy windows for the different compounds. For the spinpolarized calculations $U$ was defined as the average matrix element over the two spin channels. However, both the value of $U$ and the nearest-neighbor hopping $t$ were very similar for the two spin channels.

For $\mathrm{La}_{2} \mathrm{CuO}_{4}$ and $\mathrm{TlBa}_{2} \mathrm{CuO}_{6}$ we used the reduced structures in Refs. [27,28], while for the remaining materials we
TABLE I. Energy windows used in the Wannier function construction $(\mathrm{eV})$.

\begin{tabular}{lc}
\hline \hline Compound & Window \\
\hline $\mathrm{LaCuO}_{4}$ & $-2.5 \rightarrow 2$ \\
$\mathrm{YSr}_{2} \mathrm{Cu}_{3} \mathrm{O}_{6}$ & $-2 \rightarrow 2.2$ \\
$\mathrm{TlBa}_{2} \mathrm{CuO}_{6}$ & $-2.2 \rightarrow 3$ \\
$\mathrm{YBa}_{2} \mathrm{Cu}_{3} \mathrm{O}_{6}$ & $-2 \rightarrow 3$ \\
$\mathrm{YbBa}_{2} \mathrm{Cu}_{3} \mathrm{O}_{6}$ & $-3 \rightarrow 2$ \\
$\mathrm{HgBa}_{2} \mathrm{CuO}_{4}$ & $-2.2 \rightarrow 2$ \\
$\mathrm{NdBa}_{2} \mathrm{Cu}_{3} \mathrm{O}_{6}$ & $-3 \rightarrow 2$ \\
$\mathrm{LaBa}_{2} \mathrm{Cu}_{3} \mathrm{O}_{6}$ & $-3 \rightarrow 2$ \\
\hline \hline
\end{tabular}

used the experimental structures. The crystal structures were taken from Ref. [29] for $\mathrm{YSr}_{2} \mathrm{Cu}_{3} \mathrm{O}_{6}$, from Ref. [30] for $\mathrm{YBa}_{2} \mathrm{Cu}_{3} \mathrm{O}_{6}$, from Ref. [31] for $\mathrm{HgBa}_{2} \mathrm{CuO}_{4}$, and from [32] for $\mathrm{YbBa}_{2} \mathrm{Cu}_{3} \mathrm{O}_{6}, \mathrm{NdBa}_{2} \mathrm{Cu}_{3} \mathrm{O}_{6}$, and $\mathrm{LaBa}_{2} \mathrm{Cu}_{3} \mathrm{O}_{6}$.

\section{RESULTS AND DISCUSSION}

\section{A. Static interaction}

In the one-band Hubbard model with a static Coulomb repulsion $U$ and a nearest-neighbor hopping $t$ the only free parameter is the ratio $U / t$. Therefore, this ratio provides a good measure of the degree of local correlations.

In Fig. 2 we show the static value of $U$ as well as the ratio $U / t$ for all compounds considered in this work. $U / t$ follows an increasing trend with increasing $T_{c \text { max }}$. The smallest value of $U / t$ is approximately 6 , and the largest is approximately 9 , which implies a substantial difference in the degree of local correlations in the compounds. Since the bandwidths in most of the compounds are similar, $U$ follows the same increasing trend as $U / t$, albeit not as clearly. Hence, the trend in $U / t$ can mainly be attributed to the trend in $U$ and is not an effect of a trend in the hopping parameters. The only exception to the trend is $\mathrm{La}_{2} \mathrm{CuO}_{4}$, which has a remarkably large $U$ compared to the relatively low $T_{c}$. This suggests that $\mathrm{La}_{2} \mathrm{CuO}_{4}$, which is typically considered a prototype of a cuprate high- $T_{c}$ superconductor, actually is an exceptional case. Furthermore, assuming that the trend in $U$ implies that high- $T_{c}$ superconductivity is favored by a large on-site Coulomb repulsion, the fact that $\mathrm{La}_{2} \mathrm{CuO}_{4}$ eludes the trend implies that there are other mechanisms that hamper superconductivity in this compound. While the compounds $R(\mathrm{Ba}, \mathrm{Sr})_{2} \mathrm{Cu}_{3} \mathrm{O}_{6}$ all have similar structures with two $\mathrm{CuO}$ layers, it is interesting to note that both $\mathrm{TlBa}_{2} \mathrm{CuO}_{4}$ and $\mathrm{HgBa}_{2} \mathrm{CuO}_{4}$, which are singlelayer compounds, also follow the trend. This implies that $U / t$ indeed is an important parameter to obtain large $T_{c}$ and is at least as important as other parameters, such as the number of $\mathrm{Cu}-\mathrm{O}$ layers.

The value of $U$ depends both on the screening properties and on the shape and extent of the Wannier basis functions. The value of the bare interaction $v$, on the other hand, depends on only the shape and extent of the Wannier basis functions. More localized Wannier functions yield larger values of $v$. Since the Wannier functions are derived from the band structure, trends in $v$ can be considered band structure effects, while trends in $U$ can depend both on the band structure and on the screening process. By comparing the values of $v$ 

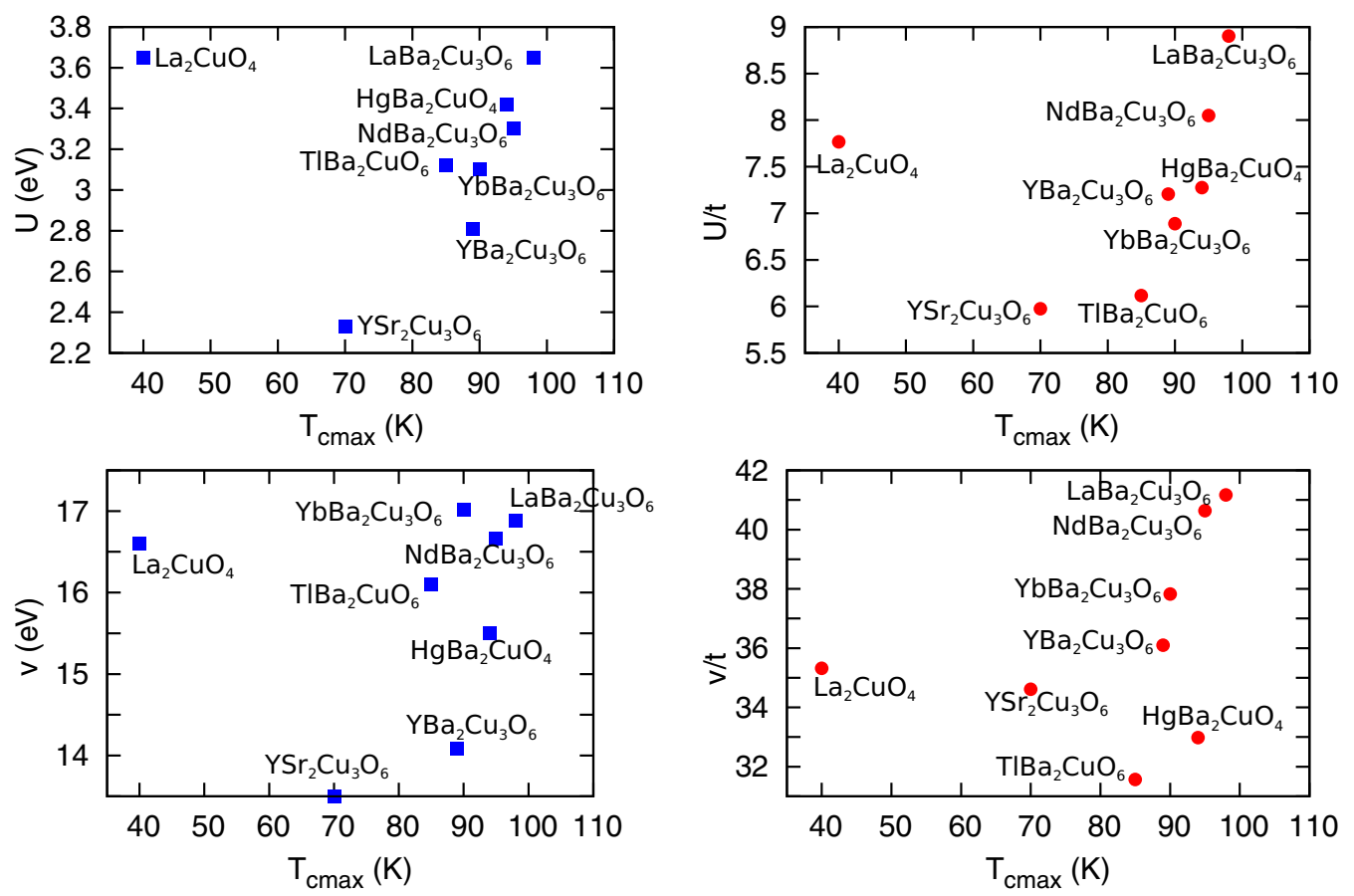

FIG. 2. Top left: Static value of $U$ for the parent compounds of a number of hole-doped cuprates in a one-band model. Top right: The ratio $U / t$ for the same compounds; $t$ is the nearest-neighbor hopping that was derived from a Wannier interpolation. Bottom left: Corresponding matrix element of the bare (unscreened) Coulomb interaction $v$. Bottom right: The ratio $v / t$.

(bottom left panel) and $U$ (top left panel) in Fig. 2 one can conclude that the trend in $U$ is intimately related to the screening in the compounds. For example, $\mathrm{TlBa}_{2} \mathrm{CuO}_{4}$ has a larger bare interaction but a smaller value of $U$ than $\mathrm{HgBa}_{2} \mathrm{CuO}_{4}$ due to the larger screening in the former compound. Also, in $\mathrm{YbBa}_{2} \mathrm{Cu}_{3} \mathrm{O}_{6}$, which has the largest value of $v$, the screening is large compared to that in the other compounds. In $v / t$ (bottom right panel of Fig. 2) the main exceptions to the trend are $\mathrm{HgBa}_{2} \mathrm{CuO}_{4}$ and $\mathrm{TlBa}_{2} \mathrm{CuO}_{4}$.

For the compound $\mathrm{YbBa}_{2} \mathrm{Cu}_{3} \mathrm{O}_{6}$ we simulated the external pressure by scaling the lattice parameters as discussed in Sec. II B. Our results are summarized in Table II. The effect of external pressure is small $(<2 \%$ for reasonable pressures $)$ on both $U$ and $U / t$. Thus, $U$ cannot be used to understand the increase of $T_{c \max }$ upon applied external pressure. It is interesting to note that, contrary to the Heisenberg exchange parameter $J$ [8], $U$ follows the same trend upon applied internal and external pressure. However, since the hopping amplitude also increases with a decreasing in-plane $\mathrm{Cu}$ distance, $U / t$ is unchanged upon applied hydrostatic pressure and decreases with in-plane pressure.

TABLE II. Effect of external pressure on $U(\mathrm{eV})$ for $\mathrm{YbBa}_{2} \mathrm{Cu}_{3} \mathrm{O}_{6}$. External pressure was simulated by reducing the lattice parameters. For in-plane pressure the $a$ lattice parameter was reduced by $1 \%$, and for hydrostatic pressure $a$ was reduced by $1 \%$, and $c$ was reduced by $2 \%$.

\begin{tabular}{lcc}
\hline \hline & $U$ & $U / t$ \\
\hline Normal & 3.1 & 6.9 \\
Hydrostatic & 3.2 & 6.9 \\
In plane & 3.2 & 6.6 \\
\hline \hline
\end{tabular}

Taken together, our results indicate that $U$ is an important parameter to get high $T_{c \text { max }}$ and that superconductivity could be favored by a large on-site Coulomb repulsion. However, there are exceptions to this trend; $\mathrm{La}_{2} \mathrm{CuO}_{4}$ has a relatively large $U$ but low $T_{c \max }$, and upon applied external pressure the change in $U$ is not sufficient to account for the observed increase in $T_{c \text { max }}$. This indicates that $U$ or $U / t$ is not the only important parameter for high- $T_{c}$ superconductivity in cuprates; rather, there seem to be many competing mechanisms that, taken together, determine whether a material has a high $T_{c}$ or not. The shape of the Fermi surface, as indicated by the trend in $T_{c \max }$ with $t^{\prime} / t$ in Ref. [1], is an example of one such important parameter.

\section{B. Comparison to other calculations}

In this section we compare our results to other similar studies. The two main studies we will focus on are the ones by Jang et al. [10] that compared $U$ for a number electron- and hole-doped cuprates and Hirayama et al. [33], who derived the low-energy Hamiltonian for $\mathrm{La}_{2} \mathrm{CuO}_{4}$ as well as $\mathrm{HgBa}_{2} \mathrm{CuO}_{4}$ in the one-, two-, and three-band models using the multi-scale ab initio scheme for correlated electrons (MACE) scheme [34-36]. We also briefly discuss the numerical studies by Weber et al. [15] and Acharya et al. [16], using cluster dynamical mean-field theory (DMFT) and ( $G W+$ DMFT)based schemes, respectively, showing a correlation between the charge-transfer energy and $T_{c}$, which indicates that superconductivity is favored by a small charge-transfer gap.

\section{Comparison to Jang et al.}

In Ref. [10] Jang et al. computed the static value of $U$ using the cRPA for the parent compounds of a number of both 
hole- and electron-doped cuprates. Of specific interest to this work they computed $U$ for $\mathrm{La}_{2} \mathrm{CuO}_{4}$ and $\mathrm{HgBa}_{2} \mathrm{CuO}_{4}$. For both these compounds they obtained a value of $U$ which is substantially smaller than the ones in this work $(3.15 \mathrm{eV}$ for $\mathrm{La}_{2} \mathrm{CuO}_{4}$ compared to $3.65 \mathrm{eV}$ in this work and $2.15 \mathrm{eV}$ for $\mathrm{HgBa}_{2} \mathrm{CuO}_{4}$ compared to $3.42 \mathrm{eV}$ in this work). The origin of this difference is the difference in methods when computing the model polarization. We used the disentanglement approach [21] described in Sec. II A, while Ref. [10] used a weighting approach [37] where the polarization is computed for the original LDA band structure and $P_{d}$ is defined as

$$
\begin{aligned}
P_{d}\left(\mathbf{r}, \mathbf{r}^{\prime} ; \omega\right)= & \sum_{\mathbf{k} n}^{\text {occ } n} \sum_{\mathbf{k}^{\prime} n^{\prime}}^{\text {unocc }}\left(\frac{\phi_{\mathbf{k}^{\prime} n}^{*}(\mathbf{r}) \phi_{\mathbf{k}^{\prime} n^{\prime}}(\mathbf{r}) \phi_{\mathbf{k}^{\prime} n^{\prime}}^{*}\left(\mathbf{r}^{\prime}\right) \phi_{\mathbf{k} n}\left(\mathbf{r}^{\prime}\right)}{\omega-\epsilon_{\mathbf{k}^{\prime} n^{\prime}}+\epsilon_{\mathbf{k} n}+i \delta}\right. \\
& \left.-\frac{\phi_{\mathbf{k} n}(\mathbf{r}) \phi_{\mathbf{k}^{\prime} n^{\prime}}^{*}(\mathbf{r}) \phi_{\mathbf{k}^{\prime} n^{\prime}}\left(\mathbf{r}^{\prime}\right) \phi_{\mathbf{k} n}^{*}\left(\mathbf{r}^{\prime}\right)}{\omega+\epsilon_{\mathbf{k}^{\prime} n^{\prime}}-\epsilon_{\mathbf{k} n}-i \delta}\right) P_{\mathbf{k} n} P_{\mathbf{k}^{\prime} n^{\prime}}
\end{aligned}
$$

$P_{\mathbf{k} n}$ is the probability that the electron in state $\left|\phi_{\mathbf{k} n}\right\rangle$ resides in the $d$ subspace. This method generally yields smaller values of $U$ since not all metallic screening from the correlated (disentangled) band is removed. Furthermore, the final aim of our calculations is to use the $U$ values together with a Hamiltonian or hopping parameters in, e.g., LDA+DMFT or $G W+$ DMFT calculations. The Hamiltonian for this type of calculation would exactly correspond to the $d$ block of the disentangled Hamiltonian in Eq. (5). Hence, in the disentanglement approach both the $U$ matrix and the hopping parameters are derived from the disentangled band structure, which is not the case in the weighting approach. We therefore consider the disentanglement approach to be a more appropriate method in this case.

In Ref. [10] the focus was on the comparison between electron-doped and hole-doped cuprates, and a general tendency of electron-doped cuprates to have a smaller $U$ was found. However, some of the hole-doped cuprates also had similarly small values of $U$, which led the authors to conclude that a correlation strong enough to induce a Mott gap may not be a prerequisite for high- $T_{c}$ superconductivity. In this work we consider a wider range of hole-doped cuprates. Even though $U$ is generally larger using the disentanglement approach $\left(U\right.$ for $\mathrm{HgBa}_{2} \mathrm{CuO}_{4}$ using the disentanglement approach is still larger than the value of $U$ computed for $\mathrm{La}_{2} \mathrm{CuO}_{4}$ with the weighting approach in Ref. [10]), at first sight, our results seem to strengthen the conclusions in Ref. [10]. $U$ for $\mathrm{YSr}_{2} \mathrm{Cu}_{3} \mathrm{O}_{6}$, for example, is only $2.33 \mathrm{eV}$, which is approximately 0.6 times the bandwidth and hence cannot be considered to be deep in the Mott-insulating regime. However, as discussed in, e.g., Ref. [9], cRPA for a pure one-band model could potentially underestimate $U$ due to the large spread of the Wannier basis states in this model. Hence, one should mainly focus on the trend rather than the absolute values of $U$ in a one-band model. The trend in the static value indicates that, even though a correlation strong enough to induce a Mott gap may or may not be a prerequisite for the high- $T_{c}$ superconductivity, superconductivity may be favored by strong local Coulomb repulsions.

Jang et al. found that the electron-doped cuprates, which have smaller $T_{c \text { max }}$ than their hole-doped counterparts, also

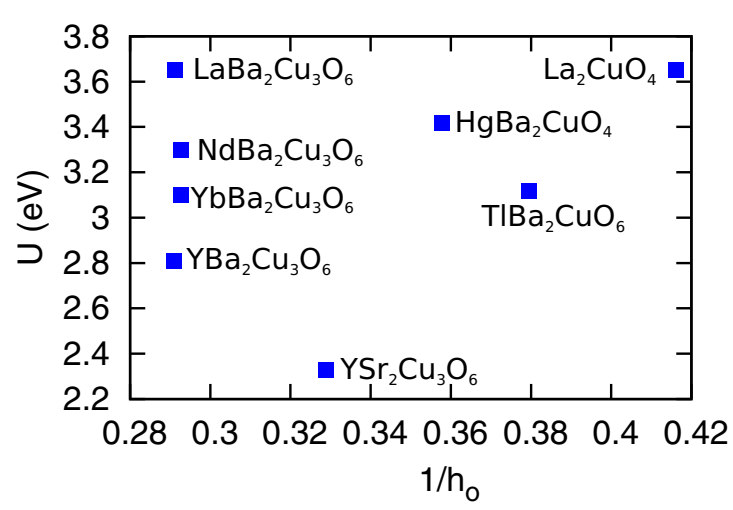

FIG. 3. Static value of $U$ as a function of the average inverse apical oxygen distance $1 / h_{O}=\frac{1}{\sqrt{2}}\left(1 / h_{O_{1}}+1 / h_{O_{2}}\right)$, where $h_{O_{1 / 2}}$ is the distance to the apical oxygen above or below the $\mathrm{Cu}$ ion in the $\mathrm{CuO}$ plane.

had smaller values of $U$. This observation fits with the trend reported in this paper. However, for $\mathrm{Hg}$-based compounds with different numbers of $\mathrm{CuO}$ layers, they also found that $U$ for the triple-layer compound $\mathrm{HgBa}_{2} \mathrm{Ca}_{2} \mathrm{Cu}_{3} \mathrm{O}_{8}$ is smaller than the corresponding values of the double- and single-layer compounds, even though $T_{c \text { max }}$ increases with the number of layers. This result contradicts the trend and therefore further illuminates the complexity of the problem with many competing mechanisms. In this particular case it points to two competing mechanisms to achieve high- $T_{c}$ superconductivity, namely, a large $U$, on the one hand, and many $\mathrm{CuO}$ layers, on the other hand.

Another interesting observation in Ref. [10] was a correlation between $U$ and the average inverse apical oxygen distance $1 / h_{O}=\frac{1}{\sqrt{2}}\left(1 / h_{O_{1}}+1 / h_{O_{2}}\right)$, where $h_{O_{1 / 2}}$ is the distance to the apical oxygen above or below the $\mathrm{Cu}$ ion in the $\mathrm{CuO}$ plane. From Fig. 3 it is clear that, while we reproduce this correlation for $\mathrm{YSr}_{2} \mathrm{Cu}_{3} \mathrm{O}_{6}, \mathrm{TlBa}_{2} \mathrm{CuO}_{6}, \mathrm{HgBa}_{2} \mathrm{CuO}_{4}$, and $\mathrm{La}_{2} \mathrm{CuO}_{4}$, the remaining compounds considered in this work do not show any correlation between $U$ and $1 / h_{O}$. For these materials the screening from the charge reservoir layers is important, as will be discussed in more detail below.

\section{Comparison to Hirayama et al.}

Hirayama et al. [33] calculated the effective low-energy Hamiltonians for $\mathrm{La}_{2} \mathrm{CuO}_{4}$ as well as $\mathrm{HgBa}_{2} \mathrm{CuO}_{4}$ in the one-, two-, and three-band models using the MACE scheme [34-36]. Of interest to this work are their values of $U$ in the one-band model $\left(5.00 \mathrm{eV}\right.$ for $\mathrm{La}_{2} \mathrm{CuO}_{4}$ and $4.37 \mathrm{eV}$ for $\mathrm{HgBa}_{2} \mathrm{CuO}_{4}$ ), which are substantially larger than the ones in this work. The MACE scheme involves a cRPA calculation using the disentanglement approach, but for the 17 bands closest to the Fermi energy (which include the $\mathrm{Cu} d$ bands and oxygen $p$ bands) they computed the polarization from the $G W$ quasiparticle band structure rather than the LDA. This yielded smaller screening and hence larger values of $U$.

The reason that $\mathrm{La}_{2} \mathrm{CuO}_{4}$ has a relatively large $U$ was also analyzed. It was concluded that $\mathrm{La}_{2} \mathrm{CuO}_{4}$ has a larger value of $U$ than $\mathrm{HgBa}_{2} \mathrm{CuO}_{4}$ because the oxygen $p$ orbitals are farther below the $\mathrm{Cu} d_{x^{2}-y^{2}}$ orbitals in $\mathrm{La}_{2} \mathrm{CuO}_{4}$, which 

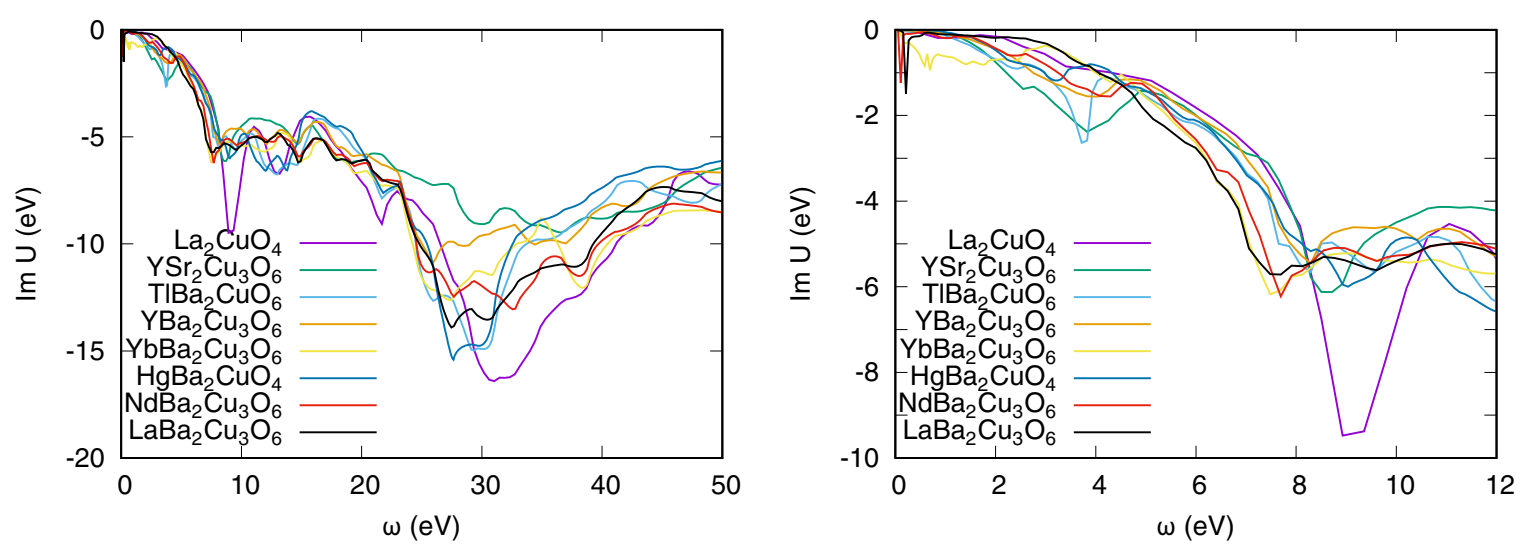

FIG. 4. Left: Imaginary part of the frequency-dependent $U$ for the different compounds. Right: Close-up of the low-frequency structure.

yields a different (more localized) character of the antibonding conduction band. This analysis would imply that both $U$ and the bare Coulomb interaction $v$ should be larger for $\mathrm{La}_{2} \mathrm{CuO}_{4}$, which also agrees with our results in Fig. 2.

\section{Comparison to Weber et al. and Acharya et al.}

In Ref. [15] Weber et al. studied $T_{c}$ as a function of the charge-transfer energy $\left(\epsilon_{d}-\epsilon_{p}\right)$ as well as the hopping parameters using cluster DMFT for the three-band model with a fixed value of $U_{d d}=8 \mathrm{eV}$. It was found that the chargetransfer energy shows an antilinear correlation with the static order parameter; that is, decreasing $\left(\epsilon_{d}-\epsilon_{p}\right)$ yielded a larger superconducting order parameter. It was also shown that the charge-transfer energy, computed from the LDA band structure, displayed an antilinear correlation with the experimental $T_{c \text { max }}$ for a large number of cuprates.

In Ref. [16] Acharya et al. studied how the displacement of the apical oxygen in $\mathrm{La}_{2} \mathrm{CuO}_{4}$ affects the superconducting order parameter, optical gap, and spin and charge susceptibilities using a one-shot combination of quasiparticle self-consistent $G W$ (QPSCGW) and DMFT. They used a static $U$ of $10 \mathrm{eV}$, which is substantially larger than the static cRPA value [9], but ignored the frequency dependence. This large $U$ value was motivated by comparing full and restricted QPSCGW calculations in Ref. [38]. It was found that pristine $\mathrm{LaCuO}_{4}$ was Mott insulating, but increasing the distance between the apical oxygen and the $\mathrm{Cu}-\mathrm{O}$ plane $\delta$ yields a crossover to a charge-transfer insulator (CTI). Increasing $\delta$ further shrinks the CTI gap, and the gap collapses at the critical value $\delta_{c}=$ 0.045 . They estimated $T_{c \max }$ from their calculated values of the superconducting order parameter and found that $T_{c \max }$ increased with increasing $\delta$ until it reached its maximum value at $\delta=\delta_{c}$. These results support the conclusions by Weber et al. that superconductivity is favored by a small chargetransfer gap.

Since an estimation of the charge-transfer gap requires the use of a three-band model, straightforward comparisons between these two works and our results are difficult. However, as discussed in detail in Ref. [9], in practical calculations for the three-band model using, e.g., LDA+DMFT, $U_{p p}$ and $U_{p d}$ are typically ignored. In such so-called $d-d p$ model calculations, the $p$ - $d$ screening should be included in the effective $U_{d d}$. Hence, the only difference between the $U_{d d}$ in the one-band model and the effective $U_{d d}$ in the $d-d p$ threeband model comes from the Wannier basis functions, which are more localized in the latter case. Therefore, it is reasonable to assume that the effective $U_{d d}$ in the three-band model will follow the same trend as in the one-band model but with larger overall values. Both Weber et al. and Acharya et al. effectively decreased the charge-transfer energy while keeping $U_{d d}$ fixed. This yields a transition from a Mott insulator to a pure chargetransfer insulator with the lower Hubbard band below the oxygen $p$ states. The same effect can be reached by keeping the charge-transfer gap fixed and increasing $U$. The results in this work combined with the studies above therefore suggest that superconductivity in the hole-doped cuprates is favored by having a CTI parent compound. This can be achieved by having a large $U$ and/or a small charge-transfer energy. Due to the large charge-transfer energy in $\mathrm{La}_{2} \mathrm{CuO}_{4}$ the lower Hubbard band is in the same energy range as the oxygen $p$ bands [9] in spite of the large $U$ value, which can explain why $\mathrm{La}_{2} \mathrm{CuO}_{4}$ does not follow the trend in Fig. 2.

\section{Frequency dependence}

In Fig. 4 we show the imaginary part of the frequencydependent $U$ for a selected number of compounds. The main features that can be observed in all materials are a subplasmonic peak around $8-9 \mathrm{eV}$ and the main bulk plasmon around $30 \mathrm{eV}$. The $8-9 \mathrm{eV}$ peak originates from screening from the oxygen $p$ bands below the Fermi energy [9]. To provide a rough estimation of the position of the peak we will consider a two-level system. The poles of the response function for a two-level system are given by

$$
\Omega_{n n^{\prime}}=\sqrt{\Delta \epsilon_{n n^{\prime}}^{2}+2 J_{n n^{\prime}} \Delta \epsilon_{n n^{\prime}}},
$$

where $\Delta \epsilon_{n n^{\prime}}$ is the energy difference between the states and $J_{n n^{\prime}}$ is the exchange interaction between the states. For $\mathrm{La}_{2} \mathrm{CuO}_{4}$ the oxygen $p$ bands are relatively far below the Fermi energy, which implies that $\Delta \epsilon_{n n^{\prime}}$ is large, and therefore, the $p-d$ peak appears at relatively high energy in $U$.

It is also interesting to note that the $p-d$ peak is much more pronounced in $\mathrm{La}_{2} \mathrm{CuO}_{4}$ than in the other compounds. 

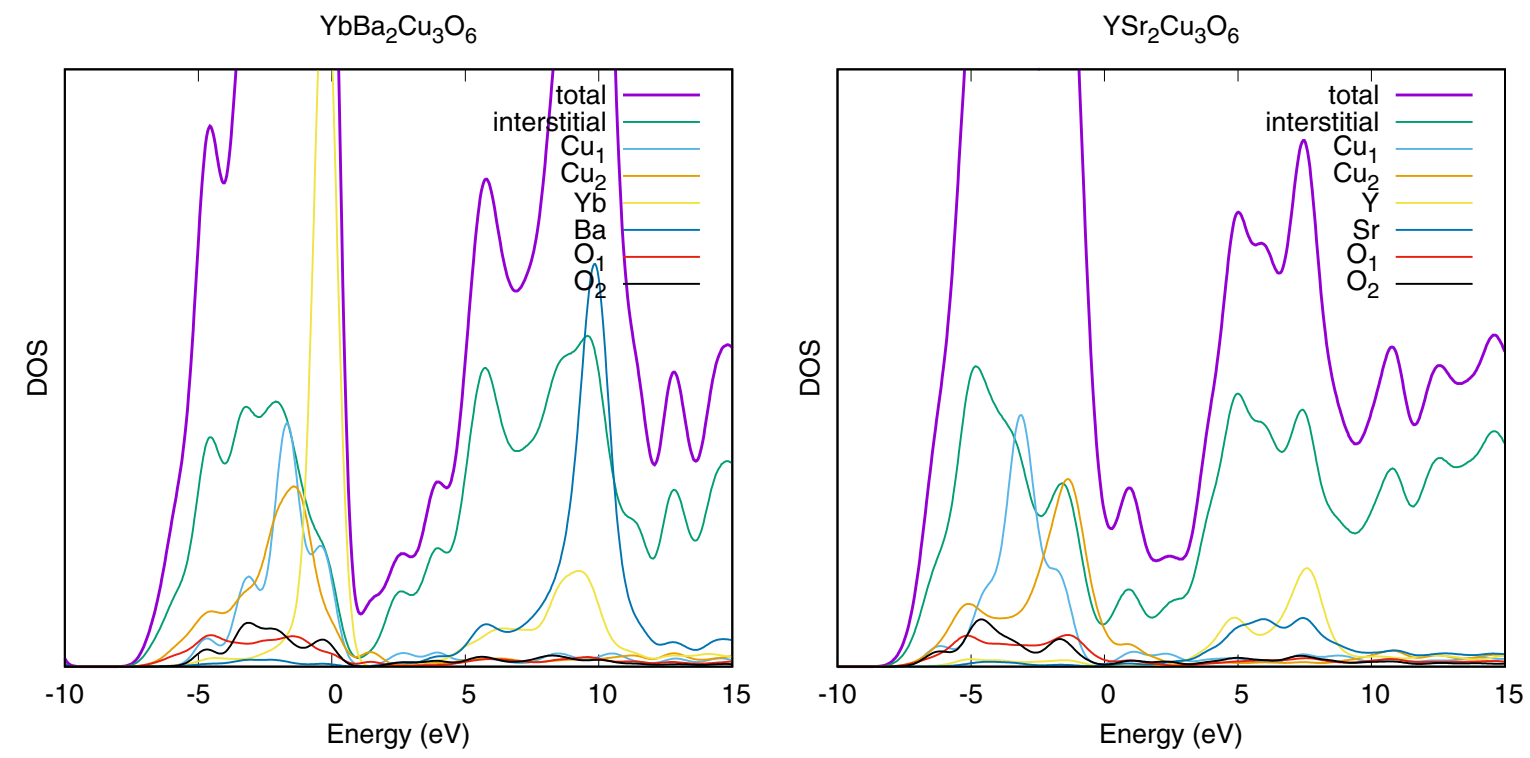

FIG. 5. Density of states (DOS) for $\mathrm{YSr}_{2} \mathrm{Cu}_{3} \mathrm{O}_{6}$ (left) and $\mathrm{YbBa}_{2} \mathrm{Cu}_{3} \mathrm{O}_{6}$ (right). The partial weights in the different muffin-tin regions are also shown. Here $\mathrm{Cu}_{2}$ is the $\mathrm{Cu}$ ion in the $\mathrm{CuO}$ plane, $\mathrm{O}_{2}$ is the apical oxygen, and $\mathrm{O}_{1}$ is the in-plane oxygen. The Fermi energy was set to zero.

If the $p$ - $d$ screening acts destructively for superconductivity, this could offer an alternative explanation of why $\mathrm{La}_{2} \mathrm{CuO}_{4}$ eludes the trend in the static $U$ in Fig. 2. However, it is possible that the large on-site Coulomb interaction, which is not accounted for when computing the cRPA $U$, suppresses the $p-d$ screening channel in the real material. The tendency of the cRPA to overestimate the low-energy screening channels between narrow bands close to the Fermi energy has also been indicated in model studies in Refs. [39-41]. The large $p$ - $d$ peak in $\mathrm{La}_{2} \mathrm{CuO}_{4}$ also indicates that the $Z$-factor renormalization [42] of the hopping elements due to the frequency dependence of the interaction is larger for $\mathrm{La}_{2} \mathrm{CuO}_{4}$ than for the other compounds.

In addition to these features the compounds with a rareearth element, $R \mathrm{Ba}_{2} \mathrm{Cu}_{3} \mathrm{O}_{6}(R=\mathrm{Yb}, \mathrm{Nd}, \mathrm{La})$, exhibit a very pronounced low-frequency structure around $0.5-1 \mathrm{eV}$. In Fig. 5 we compare the densities of states (DOSs) of $\mathrm{YSr}_{2} \mathrm{Cu}_{3} \mathrm{O}_{6}$, in which this peak is absent, and $\mathrm{YbBa}_{2} \mathrm{Cu}_{3} \mathrm{O}_{6}$, which displays the low-energy peak in $\operatorname{Im} U$. From this comparison it is tempting to conclude that the metallic screening originates from the $\mathrm{Yb}$ spectral weight close to the Fermi energy, which originates from the narrow $4 f$ band in the LDA band structure. However, these states are highly localized on the rare-earth ion and do not contribute much to the screening on the $\mathrm{Cu}$ ion. This is also apparent since $\mathrm{LaBa}_{2} \mathrm{Cu}_{3} \mathrm{O}_{6}$ does not have these $4 f$ bands but still displays a strong metallic screening. Rather, the origin of the low-energy screening channel is apparent in the band structure. In Fig. 6 the band structures of $\mathrm{LaBa}_{2} \mathrm{Cu}_{3} \mathrm{O}_{6}$ and $\mathrm{YSr}_{2} \mathrm{Cu}_{3} \mathrm{O}_{6}$ are compared. $\mathrm{LaBa}_{2} \mathrm{Cu}_{3} \mathrm{O}_{6}$ exhibits a narrow band close to the Fermi energy, which can be deduced to originate from the $d_{x z}$ and $d_{y z}$ orbitals from the out-of-plane $\mathrm{Cu}$ ion. The closeness to the Fermi energy and the strong hybridization give rise to the strong screening channel in $\operatorname{Im} U$. In $\mathrm{YSr}_{2} \mathrm{Cu}_{3} \mathrm{O}_{6}$, this band is at much lower energy and not as strongly hybridized with the $\mathrm{Cu} d_{x^{2}-y^{2}}$ conduction band.

\section{Screening analysis}

By keeping the basis functions fixed and making use of an energy window to selectively remove different screening channels it is possible to determine which screening channels contribute to the trend. Screening from all states within the energy window as well as screening due to transitions from states within the energy window to the model subspace $\left(d_{x^{2}-y^{2}}\right.$ conduction band) is removed. The bare interaction then corresponds to the case with an infinite energy window. We consider the following windows: (1) $-8 \rightarrow 2 \mathrm{eV}$ and (2) $-8 \rightarrow 12 \mathrm{eV}$. Window 1 excludes the $p-d$ screening as well as the additional low-energy screening channels, while window 2 also excludes the screening to higher-lying bands.

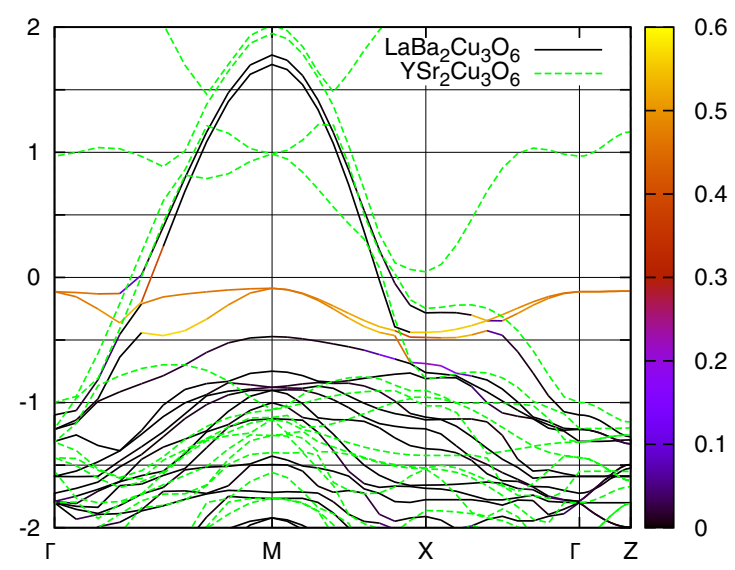

FIG. 6. LDA band structure of $\mathrm{YSr}_{2} \mathrm{Cu}_{3} \mathrm{O}_{6}$ and $\mathrm{LaBa}_{2} \mathrm{Cu}_{3} \mathrm{O}_{6}$. For $\mathrm{LaBa}_{2} \mathrm{Cu}_{3} \mathrm{O}_{6}$ we constructed maximally localized Wannier functions for the entire isolated set of 33 bands around the Fermi energy. The color coding shows the projection onto the Wannier functions centered on the out-of-plane $\mathrm{Cu}$ of $d_{x z}$ and $d_{y z}$ symmetries. The Fermi energy was set to zero. 




FIG. 7. Here we used an additional external energy window to selectively remove different screening channels in the polarization. Screening from all states within the energy window as well as screening due to transitions from states within the energy window to the model subspace $\left(d_{x^{2}-y^{2}}\right.$ conduction band) is removed. $U$ corresponds to the interaction without an additional energy window and the bare interaction, the interaction with an infinite energy window. Each data point corresponds to the ratio $U / t$ for one material with one energy window. The different values that correspond to the same energy window are connected. For a better comparison we scaled the ratio $U / t$ by its maximum value for each energy window. The order of the compounds is $\mathrm{YSr}_{2} \mathrm{Cu}_{3} \mathrm{O}_{6} \rightarrow \mathrm{TlBa}_{2} \mathrm{CuO}_{6} \rightarrow \mathrm{YBa}_{2} \mathrm{Cu}_{3} \mathrm{O}_{6} \rightarrow$ $\mathrm{YbBa}_{2} \mathrm{Cu}_{3} \mathrm{O}_{6} \rightarrow \mathrm{HgBa}_{2} \mathrm{CuO}_{4} \rightarrow \mathrm{NdBa}_{2} \mathrm{Cu}_{3} \mathrm{O}_{6} \rightarrow$ $\mathrm{LaBa}_{2} \mathrm{Cu}_{3} \mathrm{O}_{6}$.

Since we are not interested in the absolute values but only the relative trend, we show the value of $U / t$ scaled by its maximum value for each energy window in Fig. 7 . For the large energy window $(-8 \rightarrow 12 \mathrm{eV})$ the picture is almost identical to the case with the bare interaction. This shows that the high-energy screening affects all compounds in the same way and that the material-specific screening is related to the low-energy screening channels within the energy window. This can be understood from the DOSs in Fig. 5. For energies higher than $12 \mathrm{eV}$ the majority of the spectral weight comes from the interstitial region of the FLAPW basis set and therefore corresponds to broad bands that are not expected to yield a very material specific screening.

The inclusion of the screening to states between 2 and $12 \mathrm{eV}$ (see the window with $-8 \rightarrow 2 \mathrm{eV}$ ) is dramatic and highly material specific. This is expected since this energy region contains a large spectral weight on the atoms in the charge reservoir layers ( $\mathrm{Sr} / \mathrm{Ba}$, $\mathrm{Y}$, etc.), which is highly material specific. However, it is only upon the inclusion of the additional low-energy screening channels in $U$ that a clear trend can be observed. Hence, the trend cannot be attributed to any specific screening channel, but all low-energy screening channels within the $-8 \rightarrow 12 \mathrm{eV}$ energy window collectively contribute to the trend.

\section{CONCLUSIONS}

We computed the effective Coulomb interaction $U$ for the one-band model for the parent compounds of a number of hole-doped cuprate superconductors using the constrained random-phase approximation. We found a screeningdependent trend between the maximum superconducting transition temperature $T_{c \text { max }}$ and the static screened interaction $U$, suggesting that superconductivity could be favored by a large on-site effective Coulomb interaction. The only exception to the trend is $\mathrm{La}_{2} \mathrm{CuO}_{4}$, which has a relatively large value of $U$ but the smallest $T_{c \max }$. From our data we suggest that $U$ is one out of many competing parameters to achieve high$T_{c}$ superconductivity and that there are other mechanisms that hamper superconductivity in $\mathrm{La}_{2} \mathrm{CuO}_{4}$, such as the large charge-transfer energy $[15,16]$. We also studied the frequency dependence of $U$ and explained the different features. One of the most dominant features, present in all the studied compounds, is a peak in $\operatorname{Im} U(\omega)$ at around $8-9 \mathrm{eV}$, which originates from screening from the $\mathrm{O} p_{x / y}$ bands to the $d_{x^{2}-y^{2}}$ conduction band ( $p-d$ screening). This peak is much more pronounced in $\mathrm{La}_{2} \mathrm{CuO}_{4}$ than in the other compounds, which leads us to suggest that, apart from the large charge-transfer energy, the strong $p$ - $d$ screening in $\mathrm{La}_{2} \mathrm{CuO}_{4}$ could be one possible mechanism that hampers superconductivity in this compound. For the compounds $R \mathrm{Ba}_{2} \mathrm{Cu}_{3} \mathrm{O}_{6}(R=\mathrm{La}, \mathrm{Nd}, \mathrm{Yb})$, we found an additional low-energy screening channel due to screening from the band derived from the out-of-plane $\mathrm{Cu}$ $d_{x z}$ and $d_{y z}$ states. This band is close to the Fermi energy and strongly hybridized with the $d_{x^{2}-y^{2}}$ conduction band for these compounds, which yields an unusually strong screening mode.

\section{ACKNOWLEDGMENTS}

This work was supported by the Swedish Research Council (VR). The computations were performed on resources provided by the Swedish National Infrastructure for Computing (SNIC) at LUNARC. We would like to thank O. K. Andersen for useful inputs and discussions. We would also like to thank C. Friedrich and S. Blügel for providing us with their FLAPW code.
[1] E. Pavarini, I. Dasgupta, T. Saha-Dasgupta, O. Jepsen, and O. K. Andersen, Phys. Rev. Lett. 87, 047003 (2001).

[2] P. A. Lee, N. Nagaosa, and X.-G. Wen, Rev. Mod. Phys. 78, 17 (2006).

[3] H. Fukuyama and K. Yosida, Jpn. J. Appl. Phys. 26, L371 (1987).

[4] H. Yokoyama, M. Ogata, Y. Tanaka, K. Kobayashi, and H. Tsuchiura, J. Phys. Soc. Jpn. 82, 014707 (2012).
[5] J. Kaczmarczyk, J. Spałek, T. Schickling, and J. Bünemann, Phys. Rev. B 88, 115127 (2013).

[6] T. Yanagisawa, Phys. Procedia 65, 5 (2015).

[7] T. Yanagisawa, M. Miyazaki, and K. Yamaji, J. Supercond. Novel Magn. 29, 655 (2016).

[8] B. P. P. Mallett, T. Wolf, E. Gilioli, F. Licci, G. V. M. Williams, A. B. Kaiser, N. W. Ashcroft, N. Suresh, and J. L. Tallon, Phys. Rev. Lett. 111, 237001 (2013). 
[9] P. Werner, R. Sakuma, F. Nilsson, and F. Aryasetiawan, Phys. Rev. B 91, 125142 (2015).

[10] S. W. Jang, H. Sakakibara, H. Kino, T. Kotani, K. Kuroki, and M. J. Han, Sci. Rep. 6, 33397 (2016).

[11] F. Aryasetiawan, M. Imada, A. Georges, G. Kotliar, S. Biermann, and A. I. Lichtenstein, Phys. Rev. B 70, 195104 (2004).

[12] T. Miyake and F. Aryasetiawan, Phys. Rev. B 77, 085122 (2008).

[13] C. Friedrich, S. Blügel, and A. Schindlmayr, Phys. Rev. B 81, 125102 (2010).

[14] The FLEUR group, SPEX and FLEUR, http://www.flapw.de.

[15] C. Weber, C. Yee, K. Haule, and G. Kotliar, Europhys. Lett. 100, 37001 (2012).

[16] S. Acharya, C. Weber, E. Plekhanov, D. Pashov, A. Taraphder, and M. Van Schilfgaarde, Phys. Rev. X 8, 021038 (2018).

[17] W. Kohn and L. J. Sham, Phys. Rev. 140, A1133 (1965).

[18] N. Marzari and D. Vanderbilt, Phys. Rev. B 56, 12847 (1997).

[19] A. A. Mostofi, J. R. Yates, Y.-S. Lee, I. Souza, D. Vanderbilt, and N. Marzari, Comput. Phys. Commun. 178, 685 (2008).

[20] R. Sakuma, Phys. Rev. B 87, 235109 (2013).

[21] T. Miyake, F. Aryasetiawan, and M. Imada, Phys. Rev. B 80, 155134 (2009).

[22] M. Akhtar, C. Catlow, S. Clark, and W. Temmerman, J. Phys. C 21, L917 (1988).

[23] J. Schilling, in Handbook of High-Temperature Superconductivity, edited by J. Schreiffer and J. Brooks (Springer, New York, 2007), pp. 427-562.

[24] J. Schilling and S. Klotz, in Physical Properties of High Temperature Superconductors III, edited by D. M. Ginsberg (World Scientific, Singapore, 1992), pp. 59-158.

[25] A. R. Armstrong, W. I. F. David, I. Gameson, P. P. Edwards, J. J. Capponi, P. Bordet, and M. Marezio, Phys. Rev. B 52, 15551 (1995).
[26] F. Hardy, N. J. Hillier, C. Meingast, D. Colson, Y. Li, N. Barišić, G. Yu, X. Zhao, M. Greven, and J. S. Schilling, Phys. Rev. Lett. 105, 167002 (2010).

[27] A. K. McMahan, R. M. Martin, and S. Satpathy, Phys. Rev. B 38, 6650 (1988).

[28] D. J. Singh and W. E. Pickett, Physica C (Amsterdam, Neth.) 203, 193 (1992).

[29] $\mathrm{YSr}_{2} \mathrm{Cu}_{3} \mathrm{O}_{6.84}\left(\mathrm{Sr}_{2} \mathrm{Cu}_{3} \mathrm{YO}_{6.84}\right)$ crystal structure, in Pauling File Multinaries Edition - 2012, SpringerMaterials (Springer, Berlin, 2016), https://materials.springer.com/isp/ crystallographic/docs/sd_1218820.

[30] J. Swinnea and H. Steinfink, J. Mater. Res. 2, 424 (1987).

[31] S. Putilin, E. Antipov, O. Chmaissem, and M. Marezio, Nature (London) 362, 226 (1993).

[32] C. W. Chu, Proc. Natl. Acad. Sci. USA 84, 4681 (1987).

[33] M. Hirayama, Y. Yamaji, T. Misawa, and M. Imada, Phys. Rev. B 98, 134501 (2018).

[34] M. Imada and T. Miyake, J. Phys. Soc. Jpn. 79, 112001 (2010).

[35] M. Hirayama, T. Miyake, and M. Imada, Phys. Rev. B 87, 195144 (2013).

[36] M. Hirayama, T. Miyake, M. Imada, and S. Biermann, Phys. Rev. B 96, 075102 (2017).

[37] E. Şaşığlu, C. Friedrich, and S. Blügel, Phys. Rev. B 83, 121101 (2011).

[38] S. Choi, A. Kutepov, K. Haule, M. van Schilfgaarde, and G. Kotliar, npj Quantum Mater. 1, 16001 (2016).

[39] H. Shinaoka, M. Troyer, and P. Werner, Phys. Rev. B 91, 245156 (2015).

[40] Q. Han, B. Chakrabarti, and K. Haule, arXiv:1810.06116.

[41] C. Honerkamp, H. Shinaoka, F. F. Assaad, and P. Werner, Phys. Rev. B 98, 235151 (2018).

[42] M. Casula, P. Werner, L. Vaugier, F. Aryasetiawan, T. Miyake, A. J. Millis, and S. Biermann, Phys. Rev. Lett. 109, 126408 (2012). 